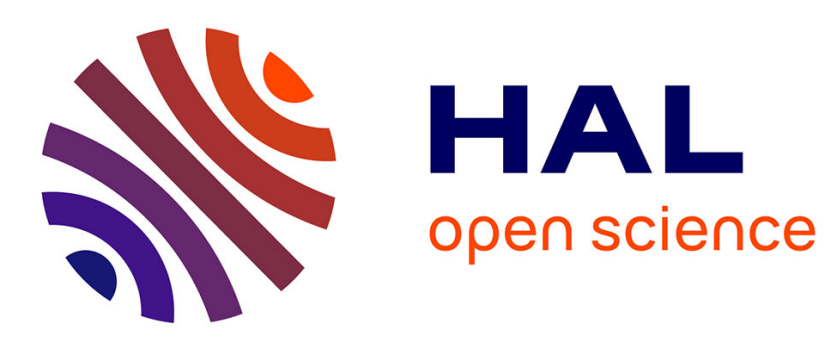

\title{
Non-symmetrical effects in the temporal bisection after selective food devaluation in rats
}

Sho Araiba, Nicole El Massioui, Bruce L. Brown, Valérie Doyère

\section{To cite this version:}

Sho Araiba, Nicole El Massioui, Bruce L. Brown, Valérie Doyère. Non-symmetrical effects in the temporal bisection after selective food devaluation in rats. Behavioural Processes, 2020, 180, pp.104244. 10.1016/j.beproc.2020.104244 . hal-02974316

\section{HAL Id: hal-02974316 https://hal.science/hal-02974316}

Submitted on 9 Dec 2020

HAL is a multi-disciplinary open access archive for the deposit and dissemination of scientific research documents, whether they are published or not. The documents may come from teaching and research institutions in France or abroad, or from public or private research centers.
L'archive ouverte pluridisciplinaire HAL, est destinée au dépôt et à la diffusion de documents scientifiques de niveau recherche, publiés ou non, émanant des établissements d'enseignement et de recherche français ou étrangers, des laboratoires publics ou privés. 
Non-symmetrical effects in the temporal bisection after selective food devaluation in rats

Sho Araiba ${ }^{1}$

Nicole El Massioui

Bruce L. Brown ${ }^{1,3}$

and

Valérie Doyère ${ }^{2}$

Graduate Center ${ }^{1}$ of the City University of New York, USA

Institut des Neurosciences Paris-Saclay (Neuro-PSI) ${ }^{2}$, UMR 9197, Université Paris Sud, CNRS,

Université Paris Saclay, Orsay, France

Queens College ${ }^{3}$, New York, USA

Corresponding author:

Sho Araiba

sho.araiba@gmail.com

1645 Ala Wai Blvd., Apt 508, Honolulu, HI 96815 


\begin{abstract}
The present study investigated the effects of selective food devaluation on performance in the temporal bisection procedure with rats. Differential outcomes (sucrose vs. grain pellets) were associated with correct responding for a short and a long duration in order to analyze the effects of a selective duration-specific food devaluation on the temporal bisection function. Selective prefeeding produced differential changes in proportion of responding, the $p$ (long) function, and PSE. A more consistent impact was observed when the food associated with the long anchor duration was devalued than when the short anchor duration food was devalued. The results are discussed in relation to the bias as well as a choose-short effect. Keywords: devaluation; prefeeding; rat; temporal bisection procedure; timing
\end{abstract}




\section{Introduction}

Interval timing behavior is often assessed using a temporal bisection procedure which evaluates discrimination of durations (Stubbs, 1976). Church and Deluty (1977) trained rats to discriminate short (S) vs. long (L) anchor durations of houselight presentations by making a different response following each duration. A test phase followed in which five geometrically spaced intermediate, unreinforced test durations were presented in addition to the reinforced $S$ and $\mathrm{L}$ anchor durations. The authors reported that the point of subjective equality (PSE) fell at the geometric mean of each set of anchor durations, the difference limen increased as the values of the anchor sets increased, and the Weber fraction remained constant.

Different types of manipulations (prefeeding, inter-trial interval food, a distractor during the timing signal, reward magnitude, stimulus presentation probability, and overtraining) can affect responses toward the short and long anchor durations, albeit differentially (Akdoğan \& Balci, 2016; Araiba et al., 2018; Cambraia et al., 2019; Es-Seddiqi et al., 2016; Galtress \& Kirkpatrick, 2010; Ward \& Odum, 2007). For example, Ward and Odum (2007) showed that prefeeding disrupted discrimination performance by decreasing responding toward the long duration sets while it did not affect responding toward the short duration stimuli. Since only one type of food was used as a reinforcer, the effect of the prefeeding manipulation could not be differentiated between responding to short and long. Even so, the resulting psychometric function did not flatten out on both ends. They interpreted this non-symmetrical effect in terms of a variant of the choose-short effect. The choose-short effect is typically observed as an increase in responding 'short' compared to responding 'long' when a delay is imposed between the duration stimulus presentation and the comparison stimuli, and may result from memory decay (e.g. Spetch \& Wikie, 1983). Ward and Odum suggested that their prefeeding manipulation, among other similar manipulations, produced the choose-short effect because of inattention of the subjects to the duration stimuli, 
resulting in the shortening of the perceived duration. Following this logic, it is conceivable that this effect might be modulated by selective prefeeding manipulation in which different types of food would be associated with each anchor duration. On the other hand, Akdoğan and Balci (2016) manipulated the probability of different anchor durations and reported a bidirectional effect on performance: an increased PSE with more short anchor trials, and a decreased PSE with more long anchor trials. Thus, selective devaluation might produce a bias effect instead of the choose-short effect. In the present study, we associated differential outcomes with correct responding for a short and a long duration in order to analyze the effects of a selective duration-specific devaluation of each food (i.e., selective satiety) on the temporal bisection function.

\section{Methods}

\subsection{Subjects}

Twenty-two adult male Sprague-Dawley rats (Harlan, France) were housed by pairs in standard laboratory cages and food restricted at $85 \%$ of their normal weight. The experiment was run in accordance with the guidelines of the European Community Council Directives of September 22nd, 2010 (2010/63/UE) and the French National Committee (2013/118) for the care and use of laboratory animals.

\subsection{Materials and procedure}

Rats were from the training group of Araiba et al. (2018), which were exposed to temporal discrimination, bisection testing, and devaluation test in extinction (see supplementary material). As pertains to the present study, rats were re-trained for two sessions to differentially respond to two levers, when presented for a maximum of $5 \mathrm{~s}$, after a 2 -s or 8 -s tone, with a correct response producing either of two different outcomes (grain vs. sucrose pellets). The rats had been divided into two groups (the experimental group and the control group), the duration/lever/food combination assignment being fully counterbalanced between 
animals within each group. The devaluation procedure consisted in three phases. First, the prefeeding phase, during which rats from the experimental group $(n=15)$ were individually given $50 \mathrm{~g}$ of either grain or sucrose pellets for an hour, corresponding to the short and long anchor reward (two devaluation conditions named Prefeed-SHORT and Prefeed-LONG). Rats from the control group $(n=7)$ were placed individually into the same prefeeding box for the same time without food. Second, immediately after prefeeding, a bisection test session was run, with 2- and 8-s anchor duration trials reinforced after correct responding, and nonreinforced trials for five intermediate test durations $(2.5,3.2,4,5$, and $6.3 \mathrm{~s})$. Third, a satiety test was run immediately following the bisection test, in which rats were presented with 50 pellets of both grain and sucrose for $5 \mathrm{~min}$ in shoe-box cages. After retraining with two sessions of the temporal discrimination, the effect of the devaluation manipulation was again tested on the bisection test, but with the other type of food used in prefeeding for a given experimental subject. This entire sequence was repeated one more time.

\subsection{Analyses}

A psychometric function for each subject was derived from the data for each of seven duration stimuli in the bisection test. PSE and gamma were estimated from the pseudologistic model (PLM, Killeen, Fetterman, \& Bizo, 1997) using GraphPad Prism 5 software (see supplementary material). Response latency less than $3 \mathrm{~s}$ was used as a criterion for inclusion in the analysis, as previously done, as long latency responses may be poorly controlled by stimulus duration (e.g., Callu et al., 2009; Es-seddiqi et al., 2016; Araiba et al., 2018). However, for comparison with some data from the literature, we also analyzed and compared bisection curves with response latencies up to $5 \mathrm{~s}$ (the maximum duration of lever presentation). Statistics were run using Jasp 0.8.2.0 with an alpha level of 0.05.

\section{Results}

3.1 Prefeeding phase and satiety test: During the prefeeding phase, rats consumed 
more sucrose $(39.5 \mathrm{~g})$ than grain $(35.8 \mathrm{~g})$ pellets, two-tailed $\mathrm{t}(14)=5.62, \mathrm{p}<.001$.

Consumption was similar for prefeed-SHORT and prefeed-LONG conditions ( $37.5 \mathrm{~g}$ vs $37.7 \mathrm{~g}$, $\mathrm{t}(14)=.14, \mathrm{p}=.89)$. During the satiety test, the effect of prefeeding, as measured by the number of pellets left of grain or sucrose, was similarly successful for each food (a main effect of prefeeding condition [46 vs 10 pellets left]: $F(1,14)=272.1$, $p<.001$, no effect of food type: $\mathrm{F}(1,14)=.41, \mathrm{p}=.53$, and no interaction: $\mathrm{F}(1,14)=.51, \mathrm{p}=.48)$, confirming selective devaluation. When analyzed by food associated with duration, the devaluation effect did not differ between durations (no effect of duration, $\mathrm{F}(1,14)=.03, \mathrm{p}=.86$, and no interaction, $\mathrm{F}(1,14)=.11, \mathrm{p}=.75)$

3.2 Bisection test: A within-subject comparison examined the effect of devaluation of short and long anchor durations for the experimental group (Figure 1A). A 2 (prefeeding condition) $\mathrm{x} 7$ (duration) ANOVA of $p$ (long) yielded main effects of prefeeding, $\mathrm{F}(1,13)=11.16, \mathrm{p}=$ .005 and duration, $\mathrm{F}(6,78)=112.02, \mathrm{p}<.001$, and a prefeeding $\mathrm{x}$ duration interaction, $\mathrm{F}(6$, $78)=3.72, p=.003$. Post-hoc analyses showed that the interaction reflected differences between conditions at only some intermediate durations, due in part to higher inter-individual variability in Prefeed-SHORT condition (Figure 1B), but not at the anchor durations.

Between-subject comparisons of the Prefeed-SHORT condition with the control group (2 [group] x 7 [duration] mixed ANOVA) yielded no main effect of group, $\mathrm{F}(1,19)=2.14$, p = .16 , a main effect of duration, $\mathrm{F}(6,114)=85.99, \mathrm{p}<.001$, and a group $\mathrm{x}$ duration interaction, $\mathrm{F}(6,114)=4.34, \mathrm{p}<.001$. For the Prefeed-LONG condition, comparisons with the control group yielded main effects of group, $\mathrm{F}(1,20)=54.31, \mathrm{p}<.001$, and duration, $\mathrm{F}(6,120)=$ $277.29, \mathrm{p}<.001$, and a group $\mathrm{x}$ duration interaction, $\mathrm{F}(6,120)=21.08, \mathrm{p}<.001$.

3.3 Level of responding: The proportion of total trials on which a response occurred was calculated for each rat at each duration in a condition (pooled across sessions). A withinsubject comparison of group mean proportion of responses as a function of duration for the 
two prefeeding conditions (Figure 1C) in a 2 (prefeeding) x 7 (duration) ANOVA yielded main effects of prefeeding, $\mathrm{F}(1,14)=6.86, \mathrm{p}=.02$, and duration, $\mathrm{F}(6,84)=9.12, \mathrm{p}<.001$, and a prefeeding $\mathrm{x}$ duration interaction, $\mathrm{F}(6,84)=6.37, \mathrm{p}<.001$. Single-duration analyses showed significant differences only at the four shorter durations. Comparisons with the control group yielded significant effects with the Prefeed-SHORT condition: main effects of group, $\mathrm{F}(1,20)=18.98, \mathrm{p}=.001$, and duration, $\mathrm{F}(6,120)=3.30, \mathrm{p}=.005$, and a group $\mathrm{x}$ duration interaction, $\mathrm{F}(6,120)=2.18, \mathrm{p}=.05$; and with the Prefeed-LONG condition: main effects of group, $\mathrm{F}(1,20)=19.09, \mathrm{p}<.001$, and duration, $\mathrm{F}(6,120)=7.66, \mathrm{p}<.001$, and a group $\mathrm{x}$ duration interaction, $\mathrm{F}(6,120)=6.71, \mathrm{p}<.001$. While the differences between short or long conditions $v s$. the control group are evident at all durations, they appear to be more pronounced at the extreme durations.

3.4 PSE and Gamma: Analyses of the PSE showed significant difference between the two devaluation conditions (Figure 1D: $\mathrm{t}(12)=3.88, \mathrm{p}=.002$ ). Prefeeding with food associated with the long anchor increased the PSE compared to the control, $\mathrm{t}(20)=6.00, \mathrm{p}<.001$, but not prefeeding with food associated with the short anchor, $\mathrm{t}(18)=1.08, \mathrm{p}=.29$. Temporal sensitivity (gamma; Figure 1E) was not significantly different between the two devaluation conditions, $\mathrm{t}<1$, and only the prefeeding with food associated with the long anchor duration increased gamma significantly in comparison with the control group, $\mathrm{t}(20)=2.97, \mathrm{p}=.008$. 3.5 Role of criterion latency: Comparison of the bisection curves calculated with response latency less than $3 \mathrm{~s}$ or less than 5s (until the limited hold) for each group/condition highlights that functions largely overlap for the control group, whereas differences appear mainly at the anchor durations in the devaluation conditions, i.e. at both short and long anchors for the Prefeed-SHORT condition, and only at the long anchor duration for the Prefeed-LONG condition (Figure 1F). 


\section{Discussion}

Devaluation by selective satiety procedure produced significant changes in proportion of responding during the bisection test, the $p$ (long) function, and PSE. Devaluation by prefeeding with rewards associated with either anchor duration had a disruptive effect on the bisection function, with a more consistent impact when the food associated with the long anchor duration was devalued, but with more variability when the food associated with the short anchor duration was devalued. Both devaluation conditions (Prefeed-SHORT and Prefeed-LONG) produced significant effects at some intermediate durations, but not at anchor durations.

In addition to selective devaluation as assessed through the satiety test, a general effect of food devaluation was seen in the proportion of responding in the experimental group compared to the control group with a decrease in responding in both prefeeding conditions, demonstrating a general (as opposed to selective) motivational effect of devaluation. In addition, we found a differential effect on the proportion of responding between the devaluation of the short and long anchor rewards with a greater decrease in the proportion of responding for the Prefeed-SHORT condition compared to that of the Prefeed-LONG condition.

During bisection testing, the $p$ (long) functions showed that the devaluation manipulation shifted the functions to the right for both the Prefeed-SHORT and the PrefeedLONG conditions compared to the control group. The devaluation of the long anchor duration yielded a reliable disruption of the bisection function (i.e. increase in PSE) and a decrease in sensitivity (i.e., increase in gamma). The devaluation of the short anchor duration had less reliable effects on temporal sensitivity, with a larger inter-individual variability potentially in part due to a lower level of responding, and no significant effect on the PSE. Finally, selective degradation of temporal stimulus control at both anchors was found following devaluation of 
short and long rewards only when including responses with longer latency, i.e. under poorer control by the stimulus duration (Figure 1F).

The present results are similar to the effects of prefeeding on the psychometric functions in Ward and Odum (2007) where non-selective prefeeding resulted in a chooseshort-like effect suggesting a possible attentional deficit. Ward and Odum suggested that the choose-short effect in the temporal bisection task might have been caused by a loss of attention due to an overall decrease in motivation. When attention to the sample cue is reduced, its perceived duration would be shorter than the remembered long anchor duration, thus producing the choose-short effect, a reduction in $p$ (long) for longer sample durations. Aspects of the present data are consistent with the choose-short effect in both experimental conditions compared to the control group (Figure 1A), and may be attributable to the general motivational effects of the devaluation manipulation reflected in the decrease in proportion of responses. Conceivably, the selective nature of the devaluation manipulation may have produced differential inattention to the two alternatives resulting in different amplitudes of shifts in $p$ (long).

Unlike the results of the selective manipulation of anchor probability in Akdogan and Balci (2016), selective devaluation did not produce symmetrical shifts (bias) in the psychometric functions when compared to the control group (see also Cambraia et al., 2019). That is, our devaluation procedure tended to produce a shift to the right or no shift (Figure 1A) instead of shifts in opposite directions from control. A possible reason for this discrepancy is that the choose-short effect associated with nonspecific food devaluation may have competed with selective bias effects in the Prefeed-SHORT condition. In any case, it appears that selective devaluation differs functionally from other selective anchor manipulations with attentional deficits dominating bias effects. 
A- Bisection curves

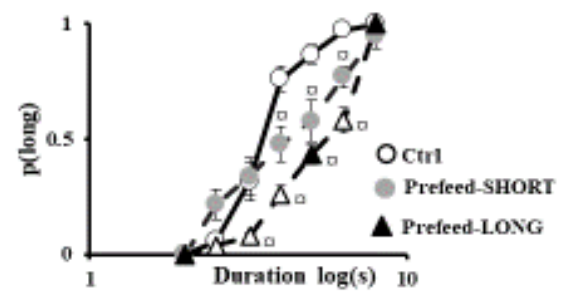

C- Level of responding

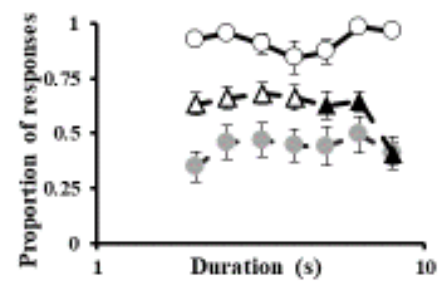

F- 5 s vs 3s comparisons

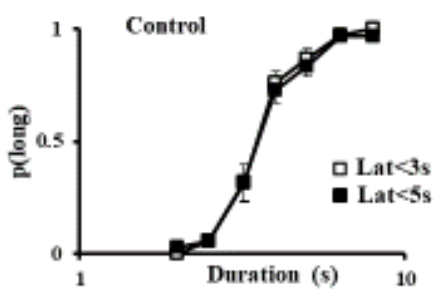

B- Individual curves
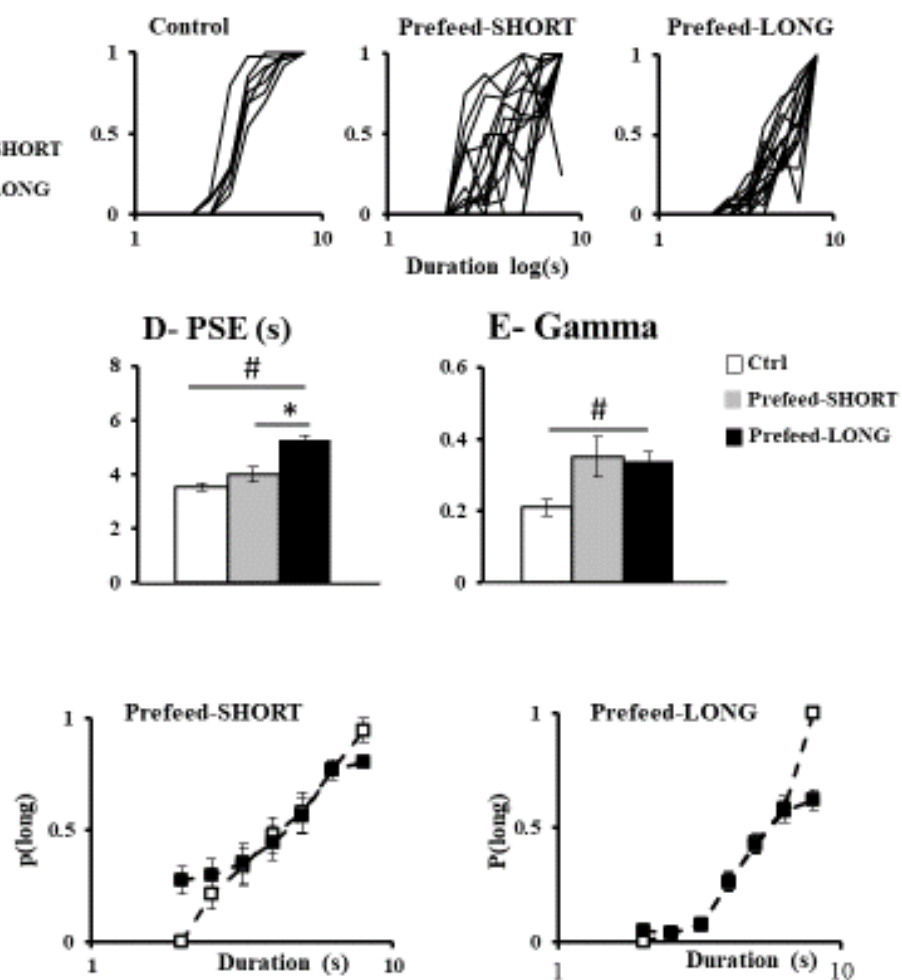

Figure 1(A): Group mean $p$ (long) as a function of duration (log scale) for the control group $(\mathrm{n}=7)$, the devaluation short (Prefeed-SHORT) condition $(\mathrm{n}=14)$, and the devaluation long (Prefeed-LONG) condition $(\mathrm{n}=15)$ (collapsed across devaluation sessions). One rat was dropped from the bisection analyses owing to no responses in the devaluation of short condition. Significant differences between Prefeed-SHORT and Prefeed-LONG curves are indicated by empty triangles at the Prefeed-LONG's durations. Symbol a above or beside certain durations of the Prefeed-SHORT and Prefeed-LONG curves indicate significant differences with the Control values. $(B)$ Individual subjects' $p$ (long) functions for the control group (left), Prefeed-SHORT condition (middle), and prefeed-LONG condition (right). $(C)$ Group mean proportion of responses as a function of duration for the control group and the experimental conditions during bisection tests. Significant differences between PrefeedSHORT and Prefeed-LONG curves are indicated by empty triangles at the Prefeed-LONG's proportions. (D) PSE for the control group and each devaluation condition. (E) Gamma for the control group and each devaluation condition. Data from one animal with $r^{2}<.57$ in the Prefeed-SHORT condition were discarded for calculation of PSE and gamma (see Supplementary material). For D and E, significant between- and within-subject comparisons are indicated by \# and * respectively. $(F)$ Comparisons of $p$ (long) functions between the 3and 5-s response latency criteria in each group/condition. Errors bars are \pm SEM. $\alpha=.05$ for significant differences. 


\section{Acknowledgements}

The authors acknowledge the support of Partner University Fund grant awarded to V. Doyère and B.L. Brown, and Agence Nationale de la Recherche awarded to V. Doyère. 


\section{Reference}

Akdoğan, B., \& Balc1, F. (2016). Stimulus probability effects on temporal bisection performance of mice (Mus musculus). Animal cognition, 19(1), 15-30. doi:10.1007/s10071-015-0909-6

Araiba, S., El Massioui, N., Brown, B. L., \& Doyère, V. (2018). Duration-specific effects of outcome devaluation in temporal control are differentially sensitive to amount of training. Learning \& Memory, 25(12), 629-633. doi:10.1101/lm.047878.118

Callu, D., El Massioui, N., Dutrieux, G., Brown, B. L., \& Doyere, V. (2009). Cognitive processing impairments in a supra-second temporal discrimination task in rats with cerebellar lesion. Neurobiology of learning and memory, 91(3), 250-259. https://doi.org/10.1016/j.nlm.2008.12.002

Cambraia, R., Vasconcelos, M., Jozefowiez, J., \& Machado, A. (2019). Biasing performance through differential payoff in a temporal bisection task. Journal of Experimental Psychology: Animal Learning and Cognition, 45(1), 75. https://doi.org/10.1037/xan0000192

Church, R. M., \& Deluty, M. Z. (1977). Bisection of temporal intervals. Journal of Experimental Psychology: Animal Behavior Processes, 3(3), 216-228. doi:10.1037/0097-7403.3.3.216

Es-Seddiqi, M., El Massioui, N., Samson, N., Brown, B. L., \& Doyère, V. (2016). The amygdalo-nigrostriatal network is critical for an optimal temporal performance. Learning \& Memory, 23(3), 104-107. doi:10.1101/lm.041152.115

Galtress, T., \& Kirkpatrick, K. (2010). Reward magnitude effects on temporal discrimination. Learning and motivation, 41(2), 108-124. https://doi.org/10.1016/j.lmot.2010.01.002 
Killeen, P., Fetterman, G., \& Bizo, L. A. (1997). Time’s causes. In: Bradshaw, C. M., \& Szabadi, E. (Eds.), Time and Behaviour: Psychological and Neurobiological Analyses. Elsevier Science Publishers, Amsterdam, pp. 79-131.

Ward, R. D., \& Odum, A. L. (2007). Disruption of temporal discrimination and the chooseshort effect. Animal Learning \& Behavior, 35(1), 60-70. https://doi.org/10.3758/BF03196075

Spetch, M. L., \& Wilkie, D. M. (1983). Subjective shortening: A model of pigeons' memory for event duration. Journal of Experimental Psychology: Animal Behavior Processes, 9(1), 14.

Stubbs, D. A. (1976). Scaling of stimulus duration by pigeons. Journal of the Experimental Analysis of Behavior, 26(1), 15-25. doi: 10.1901/jeab.1976.26-15 